TRANSACTIONS OF THE

AMERICAN MATHEMATICAL SOCIETY

Volume 356, Number 6, Pages 2307-2324

S 0002-9947(03)03278-1

Article electronically published on October 6, 2003

\title{
ON THE ADJUNCTION MAPPING OF VERY AMPLE VECTOR BUNDLES OF CORANK ONE
}

\author{
ANTONIO LANTERI, MARINO PALLESCHI, AND ANDREW J. SOMMESE
}

To the memory of Meeyoung Kim

\begin{abstract}
Let $\mathcal{E}$ be a very ample vector bundle of rank $n-1$ over a smooth complex projective variety $X$ of dimension $n \geq 3$. The structure of $(X, \mathcal{E})$ being known when $\kappa\left(K_{X}+\operatorname{det} \mathcal{E}\right) \leq 0$, we investigate the structure of the adjunction mapping when $0<\kappa\left(K_{X}+\operatorname{det} \mathcal{E}\right)<n$.
\end{abstract}

\section{INTRODUCTION}

Given a very ample line bundle $L$ on an $n$-dimensional projective manifold, $X$, the structure of the adjunction mapping of the pair $(X, L)$, i.e., the mapping associated to the complete linear system $\left|K_{X}+(n-1) L\right|$, has been a major tool in many investigations [SV], BS2]. Realizing that $(n-1) L=\operatorname{det}\left(L^{\oplus(n-1)}\right)$, it is natural to study the mapping associated to $\left|K_{X}+\operatorname{det} \mathcal{E}\right|$ where $\mathcal{E}$ is a rank- $(n-1)$, very ample vector bundle on $X$. We call this the adjunction mapping associated to $(X, \mathcal{E})$.

The structure of the mapping associated to $\left|N\left(K_{X}+\operatorname{det} \mathcal{E}\right)\right|$ for large $N>0$, follows from general results [YZ]. In this article, starting from these, we investigate the mapping associated to $\left|K_{X}+\operatorname{det} \mathcal{E}\right|$ in the situation when $0<\kappa\left(K_{X}+\operatorname{det} \mathcal{E}\right)<n$.

In Section 0 we prove a number of results useful in the sequel.

In Section 1, we prove some facts about a useful transform, which for a restricted class of fibrations lets us convert the zero sets of sections of a very ample vector bundle to a very ample divisor. Precisely, assume $\pi: X \rightarrow Y$ is a locally trivial $\mathbb{P}^{n-k}$-fibration of an $n$-dimensional projective manifold $X$ over a $k$-dimensional projective manifold $Y, k \geq 2$. Assume that $\mathcal{E}$ is a rank- $(n-k+1)$ very ample vector bundle on $X$ satisfying $\mathcal{E}_{F}=\left(\mathcal{O}_{\mathbb{P}^{n-k}}(1)\right)^{\oplus(n-k+1)}$ for any fiber $F$ of $\pi$. Then Theorem (1.4) shows that a zero set $D$ of a general section of $\mathcal{E}$ maps birationally onto a very ample divisor on $Y$. In the case $k=2$, this map is an isomorphism.

In Section 2, the adjunction mapping is studied when $\kappa\left(K_{X}+\operatorname{det} \mathcal{E}\right)=1$. It is shown that except in very restricted cases, which we describe, there is a morphism $\pi: X \rightarrow Y$ of $X$ onto a smooth projective curve $Y$ such that:

a) $\pi$ has connected fibers;

b) $K_{X}+\operatorname{det} \mathcal{E}=\pi^{*} H$, where $H$ is a very ample line bundle of the form $K_{Y}+B$, with $\operatorname{deg} B>0$.

Received by the editors July 10, 2001 and, in revised form, October 22, 2002.

2000 Mathematics Subject Classification. Primary 14F05, 14N30, 14C20; Secondary 14J40.

Key words and phrases. Vector bundle (very ample), adjunction mapping. 
In Section 3, with the help of the transform studied in Section 1, we show that, if $\kappa\left(K_{X}+\operatorname{det} \mathcal{E}\right)=2$, then there is a locally trivial $\mathbb{P}^{n-2}$-fibration $\pi: X \rightarrow Y$ over a smooth surface $Y$, such that $K_{X}+\operatorname{det} \mathcal{E}=\pi^{*}\left(K_{Y}+L\right)$, where $K_{Y}+L$ is ample and spanned, $L$ is very ample, and there exists an isomorphism of zero sets of general $s \in H^{0}(\mathcal{E})$ with some $C^{\prime} \in|L|$. The main result concerning this case is the very ampleness of $K_{Y}+L$. This fact is obtained by using Reider's theorem and the properties of the classical adjunction mapping for surfaces, except for one extremely restricted case, where $\operatorname{dim} X=3, Y$ is a Del Pezzo surface of degree 2 and $L=-2 K_{Y}$. Section 4 is devoted to ruling out this case by a sequence of steps restricting more and more the invariants of $\mathcal{E}$. This also leads to the construction of an interesting threefold described at the end of the paper.

We would like to thank the M.U.R.S.T. of the Italian Government for partial support provided in the framework of the National Research Project (Cofin 2000) "Geometry on algebraic varieties", the University of Milan, and the Duncan Chair of the University of Notre Dame for making our collaboration possible.

\section{BACKGROUND MATERIAL}

We work over the complex number field. We use the standard notation and terminology from algebraic geometry. We also follow some current abuses: we do not distinguish between vector bundles and the corresponding locally free sheaves; we use the additive notation for the tensor product of line bundles. Let $M$ be a smooth projective manifold and let $\mathcal{V}$ be a vector bundle on $M$. If $S$ is a submanifold of $M$, we put $\mathcal{V}_{S}:=i^{*} \mathcal{V}$, where $i: S \hookrightarrow M$ is the inclusion; $K_{M}$ will stand for the canonical bundle of $M$. By saying that a smooth curve $C$ is hyperelliptic we mean that it contains a $g_{2}^{1}$; this is a slightly more general definition than the usual one, since it includes the case when $C$ has genus $g(C) \leq 1$.

The following observation will be very useful in the next section.

(0.1) Remark. Let $\mathcal{V}$ be a vector bundle over a smooth projective manifold $M$. Let $\mathbb{P}(\mathcal{V})$ be the projectivization of $\mathcal{V}$ with $\xi_{\mathcal{V}}$ the tautological line bundle, and let $\pi: \mathbb{P}(\mathcal{V}) \rightarrow M$ be the projection. Under the standard identification

$$
H^{0}\left(\mathbb{P}(\mathcal{V}), \xi_{\mathcal{V}}\right) \stackrel{\cong}{\longrightarrow} H^{0}(M, \mathcal{V})
$$

sections $s$ of $\xi \mathcal{V}$ with a fiber $F_{y}:=\pi^{-1}(y)$ contained in $s^{-1}(0)$ correspond to sections $\widetilde{s}$ of $\mathcal{V}$ with $y \in \widetilde{s}^{-1}(0)$. Moreover, given such sections $s, \widetilde{s}$, a vector $\tau_{y} \in T_{M, y}$ is not tangent to $\widetilde{s}^{-1}(0)$ if and only if there exist $x \in F_{y}$ and $\tau_{x} \in T_{\mathbb{P}(\mathcal{V}), x}$ with

a) $d \pi\left(\tau_{x}\right)=\tau_{y}$

b) $s^{-1}(0)$ is not tangent to $\tau_{x}$.

To see this, let $e_{0}, \ldots, e_{r-1}$ be a basis for $\mathcal{V}$ in a neighborhood of $y$ and let $z_{0}, \ldots, z_{r-1}$ be the fiberwise homogeneous coordinates corresponding to the $e_{i}$. Then

$$
s=\sum f_{i}(m) z_{i} \mapsto \widetilde{s}=\sum f_{i}(m) e_{i}
$$

The condition that $s\left(F_{y}\right)=0$ translates into $f_{i}(y)=0$ for all $i$, which is clearly equivalent to $\widetilde{s}(y)=0$. Moreover, in this situation, fix a tangent vector $\tau_{y}$ as above and let $\tau_{x}$ be a tangent vector satisfying a). We have

$$
0 \neq d \widetilde{s}\left(\tau_{y}\right)=\sum e_{i} d f_{i}\left(\tau_{y}\right)=\sum e_{i} d f_{i}\left(d \pi \tau_{x}\right)
$$


However, the last expression maps to $d s\left(\tau_{x}\right)$ via the isomorphism induced by the standard identification.

Note that the normal bundle of $F_{y}$ in $\mathbb{P}(\mathcal{V})$ is $\pi^{*} T_{M, y}=F_{y} \times T_{M, y}$; hence the last condition in the statement is equivalent to requiring that condition b) holds for every $x \in F_{y}$ and every $\tau_{x}$ satisfying a).

Now let $X$ be a smooth projective manifold of dimension $n \geq 3$ and let $\mathcal{E}$ be a vector bundle of rank $r \geq 2$ on $X$. We say that $\mathcal{E}$ is spanned at a point $x \in X$ if the evaluation homomorphism $\Gamma(\mathcal{E}) \rightarrow \mathcal{E}_{x}$ is a surjection. We say that $\mathcal{E}$ is spanned to mean that it is spanned at every point. Let $P=\mathbb{P}(\mathcal{E})$ and let $\xi$ be the tautological line bundle of $\mathcal{E}$. We say that $\mathcal{E}$ is very ample if $\xi$ is a very ample line bundle on $P$.

We need a standard consequence of very ampleness (see also BS2, Corollary 6.6.2]).

(0.2) Lemma. Assume that $\mathcal{E}$ is a very ample vector bundle on $X$, let $x \in X$ and let $\mathfrak{m}_{x}$ be the maximal ideal sheaf of $x$. Then the sheaf $\mathcal{E} \otimes \mathfrak{m}_{x}$ is spanned away from $x$.

The following lemma is preparatory material for the next result, which will play a crucial role in Section 1.

(0.3) Lemma. Let $L$ be a very ample line bundle on a smooth projective manifold $X$ of dimension $n$ and let $Z \subset X$ be a $k$ plane with respect to $L$. If $2 k<n$, then there exists a dense Zariski open subset of $|L|$ of smooth elements $H$ containing $Z$.

Proof. Let $\mathcal{I}_{Z}$ be the ideal sheaf of $Z$ in $X$. By [BS2, Lemma 6.6.1] we know that $L \otimes \mathcal{I}_{Z}$ is spanned by global sections. Then, since $2 k<n$, [BS2, Corollary 1.7.5] applies, giving the existence of a smooth element $H \in\left|L \otimes \mathcal{I}_{Z}\right|$. Actually the proof shows that the general such element is smooth.

(0.4) Lemma. Let $X$ be a smooth projective manifold of dimension $n$ and let $\mathcal{E}$ be a very ample vector bundle of rank $r \leq n$ on $X$. For every $y \in X$ and for every non-zero tangent vector $\tau_{y} \in T_{X, y}$ there exists a section $s \in \Gamma(\mathcal{E})$ such that $s^{-1}(0)$ is smooth, containing $y$ and $\tau_{y}$ is not tangent to $s^{-1}(0)$.

Proof. Consider again $P=\mathbb{P}(\mathcal{E})$ with tautological line bundle $\xi$, and let $\pi: P \rightarrow X$ be the projection. Let $F=\pi^{-1}(y)$. Since $F$ is a $(r-1)$-plane of $P$ with respect to $\xi$ and $2(r-1)<n+r-1=\operatorname{dim} P$ by assumption, by Lemma $(0.3)$ we know that there exists a dense Zariski open subset $U$ of $\Gamma\left(\xi \otimes \mathcal{I}_{F}\right)$ of sections whose zero locus is smooth and contains $F$. Now let $\mathcal{N}_{F / P}^{*}$ be the conormal bundle of $F$ in $P$ and consider the evaluation morphism

$$
F \times \Gamma\left(\xi \otimes \mathcal{I}_{F}\right) \stackrel{\mathrm{ev}}{\longrightarrow} \mathcal{N}_{F / P}^{*} \otimes L
$$

sending any section $s \in \Gamma\left(\xi \otimes \mathcal{I}_{F}\right)$ to its differential evaluated at $x$, for every $x \in F$. Since $2(r-1)<\operatorname{dim} P$ as already observed, by BS2, Proof of Corollary (1.7.5)] we know that ev is surjective. Choose any $x \in F$ and any tangent vector $\tau_{x} \in T_{P, x}$ such that $d \pi\left(\tau_{x}\right)=\tau_{y}$. Note that $\mathcal{N}_{F / P}^{*} \otimes L \cong\left(\pi^{*} T_{X, y}^{*}\right) \otimes L$. Since $\tau_{y} \neq 0$, the evaluation on $\tau_{y}$ defines a nontrivial homomorphism $T_{X, y}^{*} \rightarrow \mathbb{C}$. Hence the evaluation on $\tau_{x}$ defines a surjection $\mathcal{N}_{F / P}^{*} \otimes L \rightarrow \mathbb{C}$. Therefore the sections whose differential vanishes along $\tau_{x}$ constitute a proper vector subspace $K$ of $\Gamma\left(\xi \otimes \mathcal{I}_{F}\right)$. Thus $U \backslash K$ is still a dense Zariski open subset, and by Remark (0.1), each of its elements gives rise under the standard isomorphism $H^{0}(\xi) \cong H^{0}(\mathcal{E})$ to a section of $\mathcal{E}$ satisfying the required conditions. 
The following lemma will come up in Section 3.

(0.5) Lemma. Let $\pi: P \rightarrow \Gamma$ be a $\mathbb{P}^{m-1}$-bundle over a smooth curve $\Gamma$ of genus $g(\Gamma) \leq 1$ and let $\mathcal{E}$ be a very ample vector bundle of rank $m$ on $P$ such that $\mathcal{E}_{F} \cong$ $\mathcal{O}_{\mathbb{P}^{m-1}}(1)^{\oplus m}$ for every fiber $F$ of $\pi$. Then

$$
c_{m}(\mathcal{E}) \geq \begin{cases}m, & \text { if } g(\Gamma)=0, \\ m+2, & \text { if } g(\Gamma)=1 .\end{cases}
$$

Proof. Write $P=\mathbb{P}(\mathcal{V})$, where $\mathcal{V}$ is a vector bundle of rank $m$ over $\Gamma$, which we can assume to be very ample up to a suitable twist. Since any quotient line bundle of $\mathcal{V}$ has positive degree, we can find a lowest degree quotient line bundle, say $\mathcal{L}$ of $\mathcal{V}$. Then, up to twisting by a suitable line bundle on $\Gamma$ we can assume that $\mathcal{L}$ has degree 1. Note that, in principle, twisting can affect the very ampleness of $\mathcal{V}$; however, since any other quotient line bundle has positive degree, $\mathcal{V}$ can still be assumed to be ample. This is obvious if $g(\Gamma)=0$, since $\mathcal{V}$ is decomposable, while it follows from [Ha, Theorem 1.3] if $g(\Gamma)=1$. Let $\xi$ be the tautological line bundle on $P$ and let $\sigma$ be the section of $P$ corresponding to the surjection $\mathcal{V} \rightarrow \mathcal{L} \rightarrow 0$. Thus $\xi_{\sigma} \cong \mathcal{L}$ under the isomorphism given by $\pi_{\mid \sigma}$. Since $\mathcal{E} \otimes \xi^{-1}$ restricts trivially to every fiber of $\pi$ we have that $\mathcal{E} \otimes \xi^{-1}=\pi^{*} \mathcal{F}$, for a suitable vector bundle $\mathcal{F}$ of rank $m$ on $\Gamma$. In particular this gives

$$
c_{1}(\mathcal{F})=c_{1}\left(\left(\pi^{*} \mathcal{F}\right)_{\sigma}\right)=c_{1}\left(\left(\mathcal{E} \otimes \xi^{-1}\right)_{\sigma}\right)=c_{1}\left(\mathcal{E}_{\sigma}\right)-m \operatorname{deg} \xi_{\sigma} .
$$

Since $\xi_{\sigma}$ has degree 1 , this gives $c_{1}(\mathcal{F})=\operatorname{deg} \mathcal{E}_{\sigma}-m$. Furthermore

$$
c_{m}(\mathcal{E})=c_{m}\left(\xi \otimes \pi^{*} \mathcal{F}\right)=\xi^{m}+\xi^{m-1} \pi^{*} c_{1}(\mathcal{F}) .
$$

Hence, by recalling the Chern-Wu relation $\xi^{m}-\pi^{*} c_{1}(\mathcal{V}) \xi^{m-1}=0$ and the computation above, we get

$$
c_{m}(\mathcal{E})=c_{1}(\mathcal{V})+c_{1}(\mathcal{F})=c_{1}(\mathcal{V})+\operatorname{deg} \mathcal{E}_{\sigma}-m .
$$

Now let $g(\Gamma)=0$. Then both $\operatorname{deg} \mathcal{V}$ and $\operatorname{deg} \mathcal{E}_{\sigma}$ are $\geq m$ in view of the ampleness, which proves the assertion. If $g(\Gamma)=1$, since $\mathcal{V}$ is ample we have $\operatorname{deg} \mathcal{V} \geq 1$ and so

$$
c_{m}(\mathcal{E}) \geq \operatorname{deg} \mathcal{E}_{\sigma}+1-m
$$

On the other hand, since $\mathcal{E}$ is very ample, by Riemann-Roch and Serre duality we have

$$
h^{0}\left(\mathcal{E}_{\sigma}\right)=\operatorname{deg} \mathcal{E}_{\sigma} .
$$

However, $\operatorname{deg} \mathcal{E}_{\sigma} \geq 2 \mathrm{rk} \mathcal{E}_{\sigma}+1=2 m+1$ [I2, Proposition 5.2]. Therefore

$$
c_{m}(\mathcal{E}) \geq 2 m+1+1-m=m+2 .
$$

We conclude this section by collecting some material which will be needed in Section 4.

(0.6) Let $\mathcal{W}$ be a vector bundle of rank 2 on a smooth complex projective manifold $M$, let $\xi=\xi_{\mathcal{W}}$ be the tautological line bundle on $P=\mathbb{P}(\mathcal{W})$, and let $p: P \rightarrow M$ be the projection. By using the Chern-Wu relation $\xi^{2}-\xi p^{*} c_{1}(\mathcal{W})+p^{*} c_{2}(\mathcal{W})=0$ one immediately gets the following useful formulas. Let $\operatorname{dim} M=2$; then

$$
\xi^{3}=c_{1}(\mathcal{W})^{2}-c_{2}(\mathcal{W})
$$


Let $\operatorname{dim} M=3$; then

$$
\xi^{4}=c_{1}(\mathcal{W})^{3}-2 c_{1}(\mathcal{W}) c_{2}(\mathcal{W})
$$

(0.7) We recall some general facts concerning indecomposable vector bundles of rank 2 and odd degree on a smooth curve $\Gamma$ of genus 1 . We denote by $\mathcal{U}$ the nontrivial extension

$$
0 \rightarrow \mathcal{O}_{\Gamma} \rightarrow \mathcal{U} \rightarrow \mathcal{O}_{\Gamma}(x) \rightarrow 0
$$

for some $x \in \Gamma$, which is unique up to isomorphisms. Recall that

$$
h^{0}(\mathcal{U} \otimes \mathcal{L})=1+2 \operatorname{deg} \mathcal{L} \quad \text { and } \quad h^{1}(\mathcal{U} \otimes \mathcal{L})=0
$$

for any line bundle $\mathcal{L}$ on $\Gamma$ of degree $\geq 0$, while $h^{0}(\mathcal{U} \otimes \mathcal{L})=0$ for any line bundle of negative degree [Ha, Lemma 1.1]. We also recall that any indecomposable rank-2 bundle of odd degree is a twist of a $\mathcal{U}$, defined by a suitable $x \in \Gamma$. Furthermore all such bundles $\mathcal{U}$ give rise to the same elliptic ruled surface $\mathbb{P}(\mathcal{U})$ and, letting $\xi$ and $f$ denote the tautological line bundle and a fiber respectively, we have $h^{i}(\xi+t f)=$ $h^{i}(\mathcal{U}(t x))$ for some $x \in \Gamma$. Hence

$$
h^{0}(\xi+t f)=2 t+1 \quad \text { and } \quad h^{1}(\xi+t f)=0 \quad \text { for } t \geq 0,
$$

by (0.7.2). Combining (0.7.2) with Serre duality also gives

$$
h^{0}(\xi-f)=h^{0}(\mathcal{U}(-x))=h^{1}\left(\mathcal{U}^{*}(x)\right)=0 .
$$

\section{A transform For CERTAin LOCALly trivial P-Fibrations}

Let $X$ and $Y$ be smooth projective manifolds of dimensions $n$ and $k \geq 2$ respectively, let $\pi: X \rightarrow Y$ be a locally trivial $\mathbb{P}^{n-k}$-fibration in the complex topology, and let $\mathcal{E}$ be a very ample vector bundle on $X$ of rank $r=n-k+1$ such that

$$
\mathcal{E}_{F} \cong \mathcal{O}_{\mathbb{P}^{n-k}}(1)^{\oplus(n-k+1)},
$$

for any fiber $F \cong \mathbb{P}^{n-k}$ of $\pi$. In particular this situation includes the case when $\pi$ is a locally trivial $\mathbb{P}^{n-k}$-fibration in the Zariski topology, which means that $X$ is the projectivization of some vector bundle on $Y$. Due to the condition above we know that $\left(K_{X}+\operatorname{det} \mathcal{E}\right)_{F}$ is trivial for any fiber $F$ of $\pi$. Hence there exists a line bundle $H$ on $Y$ such that $K_{X}+\operatorname{det} \mathcal{E}=\pi^{*} H$.

(1.1) Remark. Let things be as above, and let $F_{y}=\pi^{-1}(y)$, for $y \in Y$. Consider the following commutative diagram

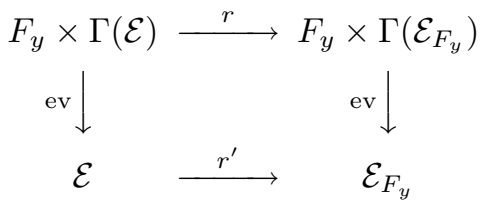

where the vertical arrows are the evaluation morphisms, hence surjective in view of the spannedness of $\mathcal{E}$, and the horizontal maps are restrictions. Note that $r^{\prime}$ is obviously surjective. Thus $r^{\prime} \circ$ ev is onto and so is the map ev $\circ r: F_{y} \times \Gamma(\mathcal{E}) \rightarrow \mathcal{E}_{F_{y}}$ for every $y \in Y$.

Note that we simply used the spannedness of $\mathcal{E}$ to get this conclusion, so the same assertion is true for $\mathcal{E}$ a merely spanned vector bundle on $X$.

Let $Z$ be the zero locus of a general section $s \in \Gamma(\mathcal{E})$. Then $Z$ is a smooth submanifold of dimension $k-1$, which is connected by the Lefschetz-Sommese theorem (see [S, Proposition 1.16] or [LM Theorem 1.1, (1.1.2)]). Let $Z^{\prime}:=\pi(Z)$. 
Note that on the general fiber $F$ of $\pi$ the section $s$ vanishes at a single point. Hence $\pi_{\mid Z}: Z \rightarrow Z^{\prime}$ is a birational morphism and an isomorphism in a neighborhood of the point lying on a general fiber. In particular $Z^{\prime}$ is an irreducible hypersurface in $Y$.

Let $Z_{i}(i=1,2)$ be the zero locus of a general section $s_{i} \in \Gamma(\mathcal{E})$, and let $\pi_{1}, \pi_{2}$ be the projections of $X \times \mathbb{P}^{1}$ onto the factors. For $(\lambda: \mu)$ varying in $\mathbb{P}^{1}$, the zero loci of the sections

$$
\lambda s_{1}+\mu s_{2} \in \Gamma\left(\pi_{2}^{*} \mathcal{O}_{\mathbb{P}^{1}}(1) \otimes \pi_{1}^{*} \mathcal{E}\right)
$$

define a $k$-dimensional variety $V$. Let $W=\left(\pi \times 1_{\mathbb{P}^{1}}\right)(V)$. Then $W$ is a family of $(k-1)$-cycles proving the rational equivalence between $Z_{1}^{\prime}=\pi\left(Z_{1}\right)$ and $Z_{2}^{\prime}=\pi\left(Z_{2}\right)$ [Fu Proposition 1.6, p. 16]. This implies that $Z_{1}^{\prime}$ and $Z_{2}^{\prime}$ are linearly equivalent, since they are divisors on a smooth projective manifold. In other words, this proves the following

(1.2) Fact. The vector bundle $\mathcal{E}$ on $X$ determines a line bundle $\mathcal{L}=\mathcal{O}_{Y}\left(Z^{\prime}\right)$ on $Y$.

Note that the line bundle $\mathcal{L}$ is spanned. Actually, let $y$ be any point on $Y$. Then the image in $\Gamma\left(\mathcal{E}_{F_{y}}\right)$ of a general section $s \in \Gamma(\mathcal{E})$ vanishes nowhere on $F_{y}$. This comes from Remark (1.1) and the fact that $\mathcal{E}_{F_{y}}$ is spanned of rank $n-k+1$, while $\operatorname{dim} F_{y}=n-k$. This means that the zero locus $Z$ of $s$ does not meet $F_{y}$. Then $Z^{\prime}=\pi(Z)$ is a divisor in $|\mathcal{L}|$ whose support does not contain $y$. This shows that the base locus of $|\mathcal{L}|$ is empty.

In fact we can prove much more. We need the following very ampleness criterion.

(1.3) Lemma. Given a line bundle $\mathcal{M}$ on a smooth projective manifold $X, \mathcal{M}$ is very ample if and only if

a) for any $x, y \in X$, with $x \neq y$, there exists an element $D \in|\mathcal{M}|$ such that $x \in D$ and $y \notin D$,

b) for all $x \in X$ and any $\tau_{x} \in T_{X, x}$ there exists an element $D \in|\mathcal{M}|$ containing $x$ and smooth at $x$ with $\tau_{x} \notin T_{D, x}$.

Proof. The conditions are obviously necessary. As to the sufficiency, spannedness of $\mathcal{M}$ and the one-to-oneness of the map $\phi$ associated to $\Gamma(\mathcal{M})$ follow from a). So to prove that $\mathcal{M}$ is very ample we need to show that $d \phi$ is injective on $T_{X, x}$. By contradiction, assume that $d \phi\left(\tau_{x}\right)=0$ for some nonzero tangent vector $\tau_{x} \in T_{X, x}$. Choose a $D \in|\mathcal{M}|$ satisfying b). Since in local coordinates the defining function $s$ for $D$ satisfies $d s\left(\tau_{x}\right) \neq 0$, it follows that $d \phi\left(\tau_{x}\right) \neq 0$.

(1.4) Theorem. The line bundle $\mathcal{L}$ is very ample.

Proof. Let $x, y \in Y$ be two distinct points and choose $x^{\prime} \in \pi^{-1}(x), y^{\prime} \in \pi^{-1}(y)$. Since $\mathcal{E}$ is very ample, by Lemma $(0.2)$ we know that $\mathcal{E} \otimes \mathfrak{m}_{x^{\prime}}$ is spanned away from $x^{\prime}$. Let $F_{y}=\pi^{-1}(y)$. Note that $\mathcal{E}_{F_{y}}=\left(\mathcal{E} \otimes \mathfrak{m}_{x^{\prime}}\right)_{F_{y}}$ since $y \neq \pi\left(x^{\prime}\right)$. So, by replacing $\mathcal{E}$ with $\mathcal{E} \otimes \mathfrak{m}_{x^{\prime}}$ and arguing as in Remark (1.1) we see that the evaluation

$$
F_{y} \times \Gamma\left(\mathcal{E} \otimes \mathfrak{m}_{x^{\prime}}\right) \rightarrow \mathcal{E}_{F_{y}}
$$

is a surjection. Since, as observed before, the general section of $\mathcal{E}_{F_{y}}$ vanishes nowhere, this shows that there is a section of $\mathcal{E} \otimes \mathfrak{m}_{x^{\prime}}$ defining a submanifold $Z$ such that $Z^{\prime}=\pi(Z)$ passes through $x$ but not through $y$. Now let $\tau_{y} \in T_{Y, y}$. We prove that there exists a divisor $Z^{\prime} \in|\mathcal{L}|$ passing through $y$, smooth at $y$, but with 
$\tau_{y}$ not tangent to it. To do that fix a point $z \in F_{y}$ and choose a tangent vector $\tau_{z} \in T_{X, x}$ such that $d \pi\left(\tau_{z}\right)=\tau_{y}$. If we show that there exists a section $s \in \Gamma(\mathcal{E})$ such that $Z=s^{-1}(0)$ is smooth containing $z$ and such that $\tau_{z}$ is not tangent to $Z$, then by taking $Z^{\prime}=\pi(Z)$ we are done. But this follows from Lemma (0.4).

\section{The CaSe $\kappa\left(K_{X}+\operatorname{det} \mathcal{E}\right) \leq 1$}

Let $X$ be a smooth complex projective variety of dimension $n \geq 3$ and let $\mathcal{E}$ be a very ample vector bundle on $X$ of rank $n-1$. Assume that the adjoint bundle $K_{X}+\operatorname{det} \mathcal{E}$ has Kodaira dimension $\kappa\left(K_{X}+\operatorname{det} \mathcal{E}\right) \geq 0$ (for the case when $\kappa\left(K_{X}+\operatorname{det} \mathcal{E}\right)=-\infty$, see [LMS]). Then, by [YZ, Theorem 3], $K_{X}+\operatorname{det} \mathcal{E}$ is nef, hence, by the Kawamata-Shokurov theorem, a suitable positive multiple of $K_{X}+\operatorname{det} \mathcal{E}$ is spanned and so defines a morphism $\varphi: X \rightarrow \mathbb{P}^{N}$. Assume that $\kappa\left(K_{X}+\operatorname{det} \mathcal{E}\right)<n$; then $\operatorname{dim} \varphi(X)<n$; let $\varphi=s \circ \pi$ be the Stein factorization, where $\pi: X \rightarrow Y$ is a morphism with connected fibers onto a normal projective variety $Y$ and $s$ is a finite morphism.

(2.1) Remark. Let things be as above; then $\operatorname{dim} Y \leq 2$.

To see this, let $F$ be a general fiber of $\pi$. Then $F$ is a submanifold of $X$ and by adjunction we get

$$
K_{F}=\left(K_{X}\right)_{F} \equiv-(\operatorname{det} \mathcal{E})_{F} .
$$

Therefore $-K_{F}$ is ample. Let $l \subset F$ be an extremal rational curve. Then $-K_{F} l \leq$ $\operatorname{dim} F+1=n+1-\operatorname{dim} Y$, by [Mo Theorem 1.2]. On the other hand

$$
-K_{F} l=(\operatorname{det} \mathcal{E}) l=c_{1}\left(\mathcal{E}_{l}\right) \geq \operatorname{rank}\left(\mathcal{E}_{l}\right)=n-1 .
$$

Thus $\operatorname{dim} Y \leq 2$.

Let $\kappa\left(K_{X}+\operatorname{det} \mathcal{E}\right)=0$. Then $Y$ is a point, hence $X=F$ is a Fano manifold and $K_{X}+\operatorname{det} \mathcal{E}=\mathcal{O}_{X}$. For the classification of pairs $(X, \mathcal{E})$ occurring in this case, see PSW.

(2.2) Now let $\kappa\left(K_{X}+\operatorname{det} \mathcal{E}\right)=1$. Then $Y$ is a smooth curve and

$$
K_{X}+\operatorname{det} \mathcal{E}=\pi^{*} H
$$

where $H$ is an ample line bundle on $Y$. Moreover, by using [BS1, Theorem 3.1] for $n=3$ and $[\mathrm{ABW}$, Theorem $\mathrm{B}$ ] for $n \geq 4$, we have the following possibilities:

(a) $X$ is a $\mathbb{P}^{n-1}$-bundle over $Y$ and $\mathcal{E}_{F}=\mathcal{O}_{\mathbb{P}^{n-1}}(2) \oplus \mathcal{O}_{\mathbb{P}^{n-1}}(1)^{\oplus(n-2)}$ for every fiber $F$ of $\pi$;

(b) $X$ is a $\mathbb{P}^{n-1}$-bundle over $Y$ and $\mathcal{E}_{F}=T_{\mathbb{P}^{n-1}}$, the tangent bundle, for every fiber $F$ of $\pi$;

(c) the general fiber $F$ of $\pi$ is isomorphic to a smooth hyperquadric $\mathbb{Q}^{n-1} \subset \mathbb{P}^{n}$ and $\mathcal{E}_{F}=\mathcal{O}_{\mathbb{Q}^{n-1}}(1)^{\oplus(n-1)}$.

Let $C$ be the the zero locus of a general section $s \in \Gamma(\mathcal{E})$. Then $C$ is a smooth curve and $p:=\pi_{\mid C}: C \rightarrow Y$ is a surjective morphism of degree $c_{n-1}\left(\mathcal{E}_{F}\right)$. Thus $\pi_{\mid C}$ is a morphism of degree 2 in cases (a) and (c) and of degree $n$ in case (b). Let $g=g(C)$ and $q=g(Y)$. Then the Riemann-Hurwitz formula gives

$$
2 g-2=(\operatorname{deg} p)(2 q-2)+\operatorname{deg} R,
$$

where $R$, the ramification divisor, has positive degree in view of the following

(2.3) Lemma. Let things be as above. Then p cannot be unbranched. 
Proof. By contradiction, assume that $p$ is unbranched and let $d \geq 2$ be its degree. Then there is a 1-cycle $\alpha$ on $Y$ whose homology class is not in $\operatorname{Im}\left(p_{*}\right)$, the image of the homomorphism $p_{*}: H_{1}(C, \mathbb{Z}) \rightarrow H_{1}(Y, \mathbb{Z})$, but such that $d \alpha \in \operatorname{Im}\left(p_{*}\right)$. Thus the cokernel of $p_{*}$ has a nontrivial $d$-torsion element. By duality we have that the cokernel of the homomorphism $p^{*}: H^{1}(Y, \mathbb{Z}) \rightarrow H^{1}(C, \mathbb{Z})$ is not torsion free. On the other hand, by the Leray spectral sequence of $\pi$ we have an isomorphism $H^{1}(X, \mathbb{Z}) \cong H^{1}(Y, \mathbb{Z})$, which, followed by $p^{*}$, gives the restriction homomorphism $\rho: H^{1}(X, \mathbb{Z}) \rightarrow H^{1}(C, \mathbb{Z})$. Thus Coker $\rho$ is not torsion free, but since $\mathcal{E}$ is ample, this contradicts the Lefschetz-Sommese theorem (see [S, Proposition 1.16] or [LM] Theorem 1.1, (1.1.2)]).

Our aim is to investigate the spannedness and the very ampleness of the line bundle $H$ appearing in (2.2.1). Our results are consistent with those obtained in [MS] for $g=2$.

(2.4) Theorem. Let $(X, \mathcal{E})$ be as in case (b). Then $H$ is spanned and it is very ample except possibly when $\operatorname{deg} H=2 q, n=3, g=3 q+1$, and either the morphism $\Phi$ defined by $\Gamma(H)$ has degree 1 , or $Y$ is hyperelliptic and $\operatorname{deg} \Phi=2$. Moreover $2 H$ is always very ample.

Proof. Let $X=\mathbb{P}(\mathcal{V})$ where $\mathcal{V}$ is an ample vector bundle on $Y$ and let $\xi$ be the tautological line bundle on $X$. Let $T_{X / Y}$ be the vertical tangent bundle of $X$. Note that $T_{X / Y} \otimes \mathcal{E}^{*}$ restricts to every fiber of $X$ as $T_{\mathbb{P}^{n-1}} \otimes T_{\mathbb{P}^{n-1}}^{*}$. On the other hand $T_{\mathbb{P}^{n-1}}$ is a simple vector bundle [OSS, Lemma 4.1.2, p. 74], hence

$$
h^{0}\left(\left(T_{X / Y} \otimes \mathcal{E}^{*}\right)_{F}\right)=1
$$

for every fiber $F$ of $X$. Therefore

$$
T_{X / Y} \cong \mathcal{E} \otimes \pi^{*} \mathcal{L},
$$

where $\mathcal{L}$ is a line bundle on $Y$. Combining this with the relative Euler sequence we get

$$
-\pi^{*} \operatorname{det} \mathcal{V}+n \xi=\operatorname{det} \mathcal{E}+(n-1) \pi^{*} \mathcal{L} .
$$

This allows us to compute $\operatorname{det} \mathcal{E}$. Adding this to the expression of $K_{X}$ given by the canonical bundle formula we get from (2.2.1)

$$
H=K_{Y}-(n-1) \mathcal{L} \text {. }
$$

Combine this with the Riemann-Hurwitz formula, recall that $\operatorname{deg} R>0$ in view of Lemma (2.3), and note that $2 g-2=n \operatorname{deg} H$ since $K_{C}=p^{*} H$. We thus conclude that $\operatorname{deg} R=n(n-1) \operatorname{deg} \mathcal{L}^{*}$. In particular $\operatorname{deg} \mathcal{L}^{*}>0$. Since $n \geq 3$, by the formula above we see that $H$ is always spanned and it is very ample except, possibly, when $n=3$ and $\operatorname{deg} \mathcal{L}^{*}=1$ (which gives $g=3 q+1$ ). In this case, by looking at the map $\Phi$ defined by $\Gamma(H)$, having $\operatorname{deg} H=2 q$ and $h^{0}(H)=q+1$, we see that either $\operatorname{deg} \Phi=1$ or $\operatorname{deg} \Phi=2$ and $Y$ is hyperelliptic. The assertion about $2 H$ follows immediately.

(2.5) Remark. Using an unpublished result of H. Maeda and A. Sommese for 3folds, it follows that $q=1$ in the exceptional case of Theorem (2.4).

(2.6) Proposition. Let $(X, \mathcal{E})$ be as in cases (a) or (c). If $H$ is not spanned, then $H=K_{Y}+[y]$ for some $y \in Y, g=2 q$ and both $C$ and $Y$ are hyperelliptic. 
Proof. Set $p=\pi_{\mid C}$. Since $p$ is a double cover, we have $R=p^{*} \mathcal{B}$ for some line bundle $\mathcal{B}$ on $Y$, with $\operatorname{deg} \mathcal{B} \geq 1$ in view of Lemma (2.3). From the equalities $p^{*} H=\left(K_{X}+\operatorname{det} \mathcal{E}\right)_{C}=K_{C}=p^{*}\left(K_{Y}+\mathcal{B}\right)$ we see that $H=K_{Y}+\mathcal{B}$. Note that if $\operatorname{deg} \mathcal{B} \geq 2$, then $H$ is spanned, having degree $\geq 2 g$. Thus if $H$ is not spanned, then $\operatorname{deg} \mathcal{B}=1$. Notice that to be spanned at a point $z \in Y$ is equivalent to

$$
\Gamma(H) \rightarrow \Gamma\left(H / H \otimes \mathfrak{m}_{z}\right) \rightarrow 0,
$$

which using Kodaira vanishing is equivalent to $H^{1}(H-z)=0$, i.e., $H^{0}(z-\mathcal{B})=0$, by Serre duality. So, if $H$ is not spanned, then $\mathcal{B}=[z]$, for some $z \in Y$. In particular we get $\operatorname{deg} R=2$ and then $g=2 q$. Moreover, the ramification locus $R$ consists of two distinct points, i.e., $R=x+y$. Hence $p^{*} \mathcal{B}=[x+y]=\left[z_{1}+z_{2}\right]=p^{*}[z]$, for some $z_{1}, z_{2} \in C$. Note that $z \notin\{p(x), p(y)\}$. Indeed if e.g., $z=p(x)$, then $p^{*} z=2 x$; so $2 x \sim x+y$, hence $x$ and $y$ would be linearly equivalent, which implies $g=0$. However, this is impossible; otherwise $q=0$ and then $\operatorname{deg} H=$ $2 q-1=-1$, contradicting the ampleness of $H$. Therefore $x+y$ and $z_{1}+z_{2}$ are two distinct linearly equivalent divisors; this shows that $C$ is hyperelliptic. Then $Y$ is hyperelliptic too.

As to the very ampleness of $H$ we have the following

(2.7) Theorem. Let $(X, \mathcal{E})$ be as in cases (a) or (c). Assume that $H$ is spanned and let $\Phi$ be the morphism associated to $\Gamma(H)$. If $H$ is not very ample, then either

(1) $\operatorname{deg} \Phi=1$ and $g=2 q \geq 6$ or $g=2 q+1$,

(2) $\operatorname{deg} \Phi=2, g=2 q+1, Y$ is hyperelliptic, and, if $q \geq 2$, then $H$ is $q$ times the hyperelliptic line bundle,

(3) $\operatorname{deg} \Phi=3$ and $g=4=2 q$.

Moreover $2 H$ is always very ample.

Proof. Let things be as in the proof of Proposition (2.6) and set $\delta=\operatorname{deg} \mathcal{B}$. Since $H=K_{Y}+\mathcal{B}$, if $H$ is not very ample we can only have $\delta \leq 2$.

Let $\delta=1$; then $g=2 q$, as before. By Riemann-Roch we have $h^{0}(H)=q \geq 2$, since $H$ is spanned. Moreover from the equality

$$
2 q-1=\operatorname{deg} H=\operatorname{deg} \Phi \operatorname{deg}(\Phi(Y))
$$

we see that $\operatorname{deg} \Phi$ is odd. Assume that $\operatorname{deg} \Phi>1$. Then $\operatorname{deg} \Phi \geq 3$. On the other hand, since $\Phi(Y)$ is a nondegenerate curve in $\mathbb{P}^{q-1}$, we have $\operatorname{deg} \Phi(Y) \geq q-1 \geq 1$; thus $3 \leq \operatorname{deg} \Phi \leq \frac{2 q-1}{q-1}$, which gives $q=2, \operatorname{deg} \Phi=3$. Now let $\operatorname{deg} \Phi=1$. Then $\Phi(Y)$ is a curve of degree $2 q-1$ in $\mathbb{P}^{q-1}$. This immediately shows that $q \geq 3$.

Let $\delta=2$. Then $\operatorname{deg} H=2 q, h^{0}(H)=q+1 \geq 2$ and $g=2 q+1$. Moreover

$$
2 q=\operatorname{deg} \Phi \operatorname{deg}(\Phi(Y)) \geq q \operatorname{deg} \Phi .
$$

Therefore, either $\operatorname{deg} \Phi=1$ or $\operatorname{deg} \Phi=2$ and $\Phi(Y)$ is a rational normal curve, hence $Y$ is hyperelliptic and if $q \geq 2$, then $H$ is $q$ times the hyperelliptic line bundle. The assertion about $2 H$ follows immediately.

\section{The CASE $\kappa\left(K_{X}+\operatorname{det} \mathcal{E}\right)=2$}

Let $\kappa\left(K_{X}+\operatorname{det} \mathcal{E}\right)=2$. Then by [BS1, Theorem 3.1] for $n=3$ and $\mathrm{ABW}$ Theorem B] for $n \geq 4, \pi: X \rightarrow Y$ is a locally trivial $\mathbb{P}^{n-2}$-fibration (in the complex topology) over a smooth surface $Y$ and $\mathcal{E}_{F} \cong \mathcal{O}_{\mathbb{P}^{n-2}}(1)^{\oplus(n-1)}$ for any fiber $F$ of $\pi$. Furthermore $K_{X}+\operatorname{det} \mathcal{E}=\pi^{*} H$, where $H$ is an ample line bundle on $Y$. 
Let $C$ again be the zero locus of a general section $s \in \Gamma(\mathcal{E})$. Then $C$ is a smooth curve. Moreover $\pi_{\mid C}: C \rightarrow Y$ is an embedding, so $C^{\prime}:=\pi(C)$ is a smooth curve, isomorphic to $C$, in $Y$. Note that $g\left(C^{\prime}\right)=g(C) \geq 2$, since

$$
2 g(C)-2=\operatorname{deg} K_{C}=\operatorname{deg}\left(K_{X}+\operatorname{det} \mathcal{E}\right)_{C}=\pi^{*} H C=H C^{\prime}>0,
$$

$H$ being ample.

By Fact (1.2) the very ample vector bundle $\mathcal{E}$ on $X$ determines a line bundle $\mathcal{L}=\mathcal{O}_{Y}\left(C^{\prime}\right)$ on $Y$, which we know to be very ample by Theorem (1.4).

In order to investigate the spannedness and the very ampleness of $H$ we prove the following

(3.1) Proposition. We have $H=K_{Y}+\mathcal{L}$.

Proof. Let $C^{\prime}=\pi(C)$ be as above. Then $C^{\prime}$ is a smooth element of $|\mathcal{L}|$. Recall that $\pi_{\mid C}: C \rightarrow C^{\prime}$ is an isomorphism. Hence, from $K_{C}=\left(K_{X}+\operatorname{det} \mathcal{E}\right)_{C}=\left(\pi^{*} H\right)_{C}$, we see that $K_{C^{\prime}}=H_{C^{\prime}}$. Thus the exact sequence

$$
0 \rightarrow-\mathcal{L} \rightarrow \mathcal{O}_{Y} \rightarrow \mathcal{O}_{C^{\prime}} \rightarrow 0
$$

twisted by $K_{Y}+\mathcal{L}-H$ gives rise to the following exact sequence:

$$
0 \rightarrow K_{Y}-H \rightarrow K_{Y}+\mathcal{L}-H \rightarrow \mathcal{O}_{C^{\prime}} \rightarrow 0 .
$$

By Serre duality combined with the Leray spectral sequence we have $H^{1}\left(K_{Y}-H\right)=$ $H^{1}(H)=H^{1}\left(K_{X}+\operatorname{det} \mathcal{E}\right)$, which is zero, by the Kodaira vanishing theorem. By taking the cohomology sequence we thus see that there exists a section of $K_{Y}+\mathcal{L}-H$ which is constant on $C^{\prime}$. Since $C^{\prime}$ is very ample by Theorem (1.4), this shows that $K_{Y}+\mathcal{L}-H \cong \mathcal{O}_{Y}$.

It is also useful to rephrase Lemma (0.5) in the present setting.

(3.2) Lemma. Let $\Gamma \subset Y$ be a smooth curve of genus $g(\Gamma) \leq 1$. Then

$$
\mathcal{L} \Gamma \geq \begin{cases}n-1, & \text { if } g(\Gamma)=0, \\ n+1, & \text { if } g(\Gamma)=1 .\end{cases}
$$

Proof. Let $C$ be as above and note that

$$
\mathcal{L} \Gamma=\pi(C) \Gamma=C \pi^{*} \Gamma=c_{n-1}\left(\mathcal{E}_{\pi^{-1}(\Gamma)}\right) .
$$

Then the assertion follows from Lemma $(0.5)$ by putting $P=\pi^{-1}(\Gamma)$.

Now we can prove

(3.3) Proposition. $H$ is spanned.

Proof. Let $C^{\prime}=\pi(C)$ be as above. Since $g\left(C^{\prime}\right) \geq 2$ and $\mathcal{L}_{C^{\prime}}$ is very ample, then $\mathcal{L}^{2} \geq 4$. If $\mathcal{L}^{2}=4$, then $C^{\prime}$ embedded by $\Gamma(\mathcal{L})$ is a canonical plane quartic; in particular $h^{0}\left(\mathcal{L}_{C^{\prime}}\right)=3$, hence $h^{0}(\mathcal{L}) \geq 4$ and indeed $=4$. Thus $\Gamma(\mathcal{L})$ maps $Y$ isomorphically to a smooth quartic surface of $\mathbb{P}^{3}$, which is a K3 surface. But in this case $H=K_{Y}+\mathcal{L}=\mathcal{L}$, so that $H$ is even very ample. Now we can assume that $\mathcal{L}^{2} \geq 5$. If $K_{Y}+\mathcal{L}$ is not spanned, then by Reider's theorem [Re], $Y$ contains an effective divisor $\Gamma$ satisfying $\mathcal{L} \Gamma=1$ (due to the ampleness). Since $\mathcal{L}$ is very ample this implies that $\Gamma$ is a smooth rational curve, but this gives a contradiction by Lemma (3.2). 
The main result on case $\kappa\left(K_{X}+\operatorname{det} \mathcal{E}\right)=2$ is the following

(3.4) Theorem. $H$ is very ample.

The proof takes the remainder of this section and Section 4. Let us consider the following special case:

(*) $n=3, X=\mathbb{P}(\mathcal{V})$ for a vector bundle $\mathcal{V}$ of rank 2 on $Y$, where $Y$ is the Del Pezzo surface with $K_{Y}^{2}=2$, and $\mathcal{E}$ is a very ample vector bundle of rank 2 on $X$ such that $\mathcal{E}_{f} \cong \mathcal{O}_{\mathbb{P}^{1}}(1)^{\oplus 2}$ for every fiber $f$ of the projection $\pi: X \rightarrow Y$, and $K_{X}+\operatorname{det} \mathcal{E}=\pi^{*}\left(-K_{Y}\right)$.

Note that in view of Proposition (3.1), the last condition in $(*)$ is equivalent to saying that $\mathcal{L}=-2 K_{Y}$. Section 4 will be devoted to proving that the assumption that $\mathcal{E}$ is very ample in $(*)$ leads to a contradiction. So Theorem (3.4) will follow from the following

(3.5) Proposition. Suppose that $X, Y, \mathcal{E}$ are not as in $(*)$. Then $H$ is very ample.

Proof. First assume that $\mathcal{L}^{2} \geq 9$. Since $\mathcal{L}$ is very ample, if $K_{Y}+\mathcal{L}$ is not very ample, then by Reider's theorem $Y$ contains an effective divisor $\Gamma$ such that either

i) $\mathcal{L} \Gamma=1$

ii) $\mathcal{L} \Gamma=2$ with $\Gamma^{2}=0$, or

iii) $\mathcal{L}=3 \Gamma$, up to numerical equivalence, and $\Gamma^{2}=1$.

In case $\mathrm{i}$ ), since $\mathcal{L}$ is very ample, $\Gamma$ is a smooth rational curve, but this contradicts Lemma (3.2). In case ii) if $\Gamma$ is reducible, it consists of two smooth rational curves of degree 1 with respect to $\mathcal{L}$, which again contradicts Lemma (3.2). If $\Gamma$ is irreducible, then it is a smooth rational curve since the map given by $\Gamma(\mathcal{L})$ is an embedding. So by the genus formula we get $\Gamma K_{Y}=-2-\Gamma^{2}=-2$; hence $H \Gamma=\left(K_{Y}+\mathcal{L}\right) \Gamma=0$, which contradicts the ampleness of $H$. In case iii) $\Gamma$ is an ample divisor, hence it is an irreducible curve, since $\Gamma^{2}=1$. Since $H$ is ample we have $\left(K_{Y}+\mathcal{L}\right) \Gamma=H \Gamma \geq 1$; hence $K_{Y} \Gamma \geq-2$, which, combined with genus formula, gives $g(\Gamma) \geq 1$. On the other hand $g(\Gamma) \leq 1$ since $\Gamma(\mathcal{L})$ maps $\Gamma$ isomorphically onto a cubic curve. Therefore $(Y, \Gamma)$ is a polarized surface of sectional genus 1 , and so we conclude from [LP. Corollary 2.4] that either

a) $Y$ is a Del Pezzo surface with $K_{Y}^{2}=1$ and $\mathcal{L}=-3 K_{Y}$, or

b) $Y=\mathbb{P}_{B}(\mathcal{U})$, where $B$ is an elliptic curve, $\mathcal{U}$ is a rank- 2 vector bundle on $B$ as in (0.7.1), and $\mathcal{L}=3 \xi, \xi$ denoting the tautological line bundle on $Y$.

In both cases $Y$ contains a smooth elliptic curve $\Gamma$ satisfying $\mathcal{L} \Gamma=3$, but this again contradicts Lemma (3.2). We can thus assume that $\mathcal{L}^{2} \leq 8$. Note that the adjunction mapping of $(Y, \mathcal{L})$ has a 2-dimensional image, since $H=K_{Y}+\mathcal{L}$ is ample. Moreover $(Y, \mathcal{L})$ cannot contain $(-1)$-lines, in view of Lemma $(3.2)$; in other words $(Y, \mathcal{L})$ coincides with its reduction. Assume that $H$ is not very ample. Then, by SV], Theorem, p. 593] we conclude that $Y$ is the Del Pezzo surface with $K_{Y}^{2}=2$ and $\mathcal{L}=-2 K_{Y}$. Let $\Gamma \in\left|-K_{Y}\right|$ be a smooth element. Since $\Gamma$ is an elliptic curve and $\Gamma \mathcal{L}=2 K_{Y}^{2}=4$, we see from Lemma (3.2) that $n \leq 3$, hence $n=3$. Moreover, since $Y$ is a rational surface, the locally trivial $\mathbb{P}^{1}$-fibration $X$ is the projectivization of a vector bundle $\mathcal{V}$ of rank 2 on $Y$, i. e. $X=\mathbb{P}(\mathcal{V})$. Therefore, $X, Y, \mathcal{E}$ are as in case $(*)$, a contradiction. 


\section{Ruling OUt CASE $(*)$}

This section is devoted to disproving the existence of case $(*)$; so assume that $X, Y, \pi, \mathcal{E}$ are as in $(*)$. We will contradict the very ampleness of $\mathcal{E}$ in several steps.

Recall that $(\operatorname{det} \mathcal{E}) f=2$ for every fiber $f$ of $\pi$, since $\mathcal{E}_{f}=\mathcal{O}_{\mathbb{P}^{1}}(1)^{\oplus 2}$. For any smooth curve $E \subset Y$ we set $X_{E}:=\pi^{-1}(E)$.

(4.1) Lemma. For every smooth curve $E \in\left|-K_{Y}\right|$ we have $X_{E}=\mathbb{P}(\mathcal{U})$, where $\mathcal{U}$ is a rank-2 bundle on $E$ as in (0.7.1). Moreover there is an exact sequence

$$
0 \rightarrow[\xi+f] \rightarrow \mathcal{E}_{X_{E}} \rightarrow[\xi+2 f] \rightarrow 0
$$

where $\xi$ is the tautological line bundle of $\mathcal{U}$ and $f$ is some fiber of $X_{E}$.

Proof. By adjunction and recalling $(*)$ we have

$$
K_{X_{E}}+\operatorname{det} \mathcal{E}_{X_{E}}=\left(K_{X}+\operatorname{det} \mathcal{E}\right)_{X_{E}}+\pi_{\mid X_{E}}^{*}\left(-K_{Y}\right)=\pi_{\mid X_{E}}^{*}\left(-2 K_{Y}\right) .
$$

Of course $X_{E}=\mathbb{P}(\mathcal{W})$ for some vector bundle $\mathcal{W}$ on $E$. If $X_{E} \neq \mathbb{P}(\mathcal{U})$, with $\mathcal{U}$ as in (0.7.1), then there are two possibilities, in view of the classification of rank-2 vector bundles on an elliptic curve; namely, up to a twist by a line bundle, either

i) $\mathcal{W}=\mathcal{O}_{E} \oplus \mathcal{L}$, where $\mathcal{L}$ is a line bundle on $E$ with $\operatorname{deg} \mathcal{L} \geq 0$, or

ii) $\mathcal{W}$ is a non-split extension of $\mathcal{O}_{E}$ by $\mathcal{O}_{E}$.

In both cases let $\xi=\xi_{\mathcal{W}}$ be the tautological line bundle on $X_{E}$ and let $f$ be a fiber. In case i) let $\sigma$ be the section associated to the surjection $\mathcal{W} \rightarrow \mathcal{O}_{E}$ onto the first summand. Since $\mathcal{E}_{f}=\mathcal{O}_{\mathbb{P}^{1}}(1)^{\oplus 2}$, up to numerical equivalence we have that $\operatorname{det} \mathcal{E}_{X_{E}}=2 \xi+b f$ for some integer $b$. However, $\operatorname{deg} \mathcal{E}_{\sigma} \geq 5$, since $\mathcal{E}$ is very ample, and this gives $b \geq 5$, recalling that $\xi \sigma=0$. We also have $K_{X_{E}}=-2 \xi+(\operatorname{deg} \mathcal{L}) f$, up to numerical equivalence. Summing up and recalling (4.1.2) we thus get

$$
5 \leq b+\operatorname{deg} \mathcal{L}=-2 K_{Y} E=4
$$

a contradiction. In case ii) we get a similar contradiction arguing in the same way. So $X_{E}=\mathbb{P}(\mathcal{U})$, where $\mathcal{U}$ is defined by a nonsplit exact sequence

$$
0 \rightarrow \mathcal{O}_{E} \rightarrow \mathcal{U} \rightarrow \mathcal{O}_{E}(x) \rightarrow 0
$$

for some $x \in E$. Let $\xi$ be the tautological line bundle on $X_{E}$. Up to numerical equivalence we have $-K_{X_{E}}=2 \xi-f$ and $\pi_{\mid X_{E}}^{*}\left(-2 K_{Y}\right)=4 f$. Hence we get $\operatorname{det} \mathcal{E}_{X_{E}}=2 \xi+3 f$, up to numerical equivalence. Let $\sigma$ be the zero set of the nontrivial section of $\xi$. Then $\operatorname{deg} \mathcal{E}_{\sigma}=(\operatorname{det} \mathcal{E})_{X_{E}} \sigma=(2 \xi+3 f) \xi=5$. This shows that $\mathcal{E}_{\sigma}$ is indecomposable. Otherwise it should have degree $\geq 6$, being the sum of two very ample line bundles on the elliptic curve $\sigma$. Since any nonsplit odd degree rank-2 bundle on an elliptic curve is a twist of $\mathcal{U}$, we see that $\mathcal{E}_{\sigma}$ is a nontrivial extension of the form

$$
0 \rightarrow \mathcal{M} \rightarrow \mathcal{E}_{\sigma} \rightarrow \mathcal{N} \rightarrow 0
$$

where $\mathcal{M}$ and $\mathcal{N}$ are line bundles on $\sigma$ of degree 2 and 3 respectively. This shows that $\mathcal{E}(-\xi)_{\sigma}=\pi_{\mid X_{E}}^{*} \mathcal{U}(y)$ for some $y \in E$. On the other hand, since $\mathcal{E}_{X_{E}}(-\xi)=$ $\pi_{\mid X_{E}}^{*} \mathcal{W}$ for some indecomposable rank-2 vector bundle $\mathcal{W}$ on $E$, in view of the isomorphism between $\sigma$ and $E$ induced by the projection, we see that $\mathcal{W} \cong \mathcal{U}(y)$. Hence

$$
\mathcal{E}_{X_{E}}=\left(\pi_{\mid X_{E}}^{*} \mathcal{U}\right) \otimes[\xi+f] .
$$

Now, pulling back (4.1.3) to $X_{E}$ and twisting by $[\xi+f]$, (4.1.4) shows that we have an exact sequence as in (4.1.1). 
(4.2) Corollary. Let $E \in\left|-K_{Y}\right|$ be any smooth curve. Then $\left(\operatorname{det} \mathcal{E}_{X_{E}}\right)^{2}=16$, $c_{2}\left(\mathcal{E}_{X_{E}}\right)=4$, and $h^{0}\left(\mathcal{E}_{X_{E}}\right)=8$.

Proof. The exact sequence (4.1.1) shows that $\left(\operatorname{det} \mathcal{E}_{X_{E}}\right)^{2}=(2 \xi+3 f)^{2}=16$ and $c_{2}\left(\mathcal{E}_{X_{E}}\right)=(\xi+f)(\xi+2 f)=4$. Moreover $h^{1}(\xi+f)=0$ by (0.7.3). Thus the cohomology sequence induced by (4.1.1) combined with (0.7.3) shows that $h^{0}\left(\mathcal{E}_{X_{E}}\right)=h^{0}(\xi+f)+h^{0}(\xi+2 f)=8$.

Recall that the surface $Y$ is $\mathbb{P}^{2}$ blown-up at seven points in general position.

(4.3) Lemma. For every exceptional curve $E \subset Y$ we have $X_{E}=\mathbb{P}\left(\mathcal{O}_{\mathbb{P 1}}^{\oplus 2}\right)$. Moreover $\mathcal{E}_{X_{E}}=\left(\mathcal{O}_{\mathbb{P}^{1} \times \mathbb{P}^{1}}(1,1)\right)^{\oplus 2}$.

Proof. Since $\left(E-K_{Y}\right)_{E}=\mathcal{O}_{E}$, by adjunction we get

$$
\begin{aligned}
K_{X_{E}}+\operatorname{det} \mathcal{E}_{X_{E}} & =\left(K_{X}+\operatorname{det} \mathcal{E}\right)_{X_{E}}+\pi_{\mid X_{E}}^{*} E \\
& =\pi_{\mid X_{E}}^{*}\left(E-K_{Y}\right)=\pi_{\mid X_{E}}^{*} \mathcal{O}_{E} \\
& =\mathcal{O}_{X_{E}} .
\end{aligned}
$$

This shows that $X_{E}$ is Fano, hence it is either $\mathbb{P}^{1} \times \mathbb{P}^{1}$ or the Segre-Hirzebruch surface $\mathbb{F}_{1}$. But in the latter case $X_{E}$ would contain a $(-1)$-curve $l$. Hence $1=$ $l\left(-K_{X_{E}}\right)=\left(\operatorname{det} \mathcal{E}_{X_{E}}\right) l=\operatorname{deg} \mathcal{E}_{l} \geq \operatorname{rk} \mathcal{E}=2$, a contradiction. Hence $X_{E}=\mathbb{P}^{1} \times$ $\mathbb{P}^{1}$. Now, since $\operatorname{det} \mathcal{E}_{X_{E}}=-K_{\mathbb{P}^{1} \times \mathbb{P}^{1}}=\mathcal{O}_{\mathbb{P}^{1} \times \mathbb{P}^{1}}(2,2)$, it is easy to see that $\mathcal{E}_{X_{E}}=$ $\left(\mathcal{O}_{\mathbb{P}^{1} \times \mathbb{P}^{1}}(1,1)\right)^{\oplus 2}$.

(4.4) Lemma. Let $X, Y, \mathcal{E}$ be as in $(*)$ and let $H$ be the tautological line bundle on $P:=\mathbb{P}(\mathcal{E})$. Then $h^{0}\left(H_{Z}\right) \geq 8$ for every smooth element $Z \in|H|$. In particular $h^{0}(\mathcal{E}) \geq 9$.

Proof. Assume, by contradiction, that $h^{0}\left(H_{Z}\right) \leq 7$. Then $\left(Z, H_{Z}\right)$ has to satisfy the double point formula for smooth threefolds embedded in $\mathbb{P}^{6}$ BS3, Theorem $0.5 .1)]$, i.e. equality must hold in the following inequality:

$$
e(Z)-48 \chi\left(\mathcal{O}_{Z}\right)+84 \chi\left(\mathcal{O}_{S}\right) \geq 11 d_{2}+17 d_{1}+d_{3}+(20-d) d,
$$

where $e$ stands for the topological Euler-Poincaré characteristic, $S$ is a smooth element of $\left|H_{Z}\right|, d=H_{Z}^{3}=H^{4}$, and the pluridegrees $d_{i}$ for $i=1,2,3$ are defined as follows:

$$
d_{1}=K_{S} H_{S}, \quad d_{2}=K_{S}^{2}, \quad d_{3}=\left(K_{Z}+H_{Z}\right)^{3} .
$$

Let us compute all the invariants involved in the formula. First note that $Z$ is simply $X$ blown-up along the zero locus of a general section of $\mathcal{E}$, which is our original curve $C$. Recall that $C$ has genus 3 and that $e(Y)=10$. Hence $e(Z)=$ $e(X)+e\left(\mathbb{P}^{1}\right) e(C)-e(C)=e(X)+e(C)=e(Y) e\left(\mathbb{P}^{1}\right)+e(C)=20-4=16$. Moreover $Z$ is birational to $X$, which is rational, hence $\chi\left(\mathcal{O}_{Z}\right)=1$. Now let $p: P \rightarrow X$ be the projection and set $q=\pi \circ p$. Since $K_{P}=-2 H+p^{*}\left(K_{X}+\operatorname{det} \mathcal{E}\right)=-2 H+q^{*}\left(-K_{Y}\right)$, by adjunction we get $K_{Z}+H_{Z}=q_{\mid Z}^{*}\left(-K_{Y}\right)$, hence $d_{3}=\left(K_{Z}+H_{Z}\right)^{3}=0$. We also have $K_{S}=\left(K_{P}+2 H\right)_{S}=q_{\mid S}^{*}\left(-K_{Y}\right)$. Notice that $q_{\mid S}: S \rightarrow Y$ is a double cover; actually since $\mathbb{P}\left(\mathcal{E}_{f}\right)=\mathbb{P}\left(\mathcal{O}_{\mathbb{P}^{1}}(1) \oplus \mathcal{O}_{\mathbb{P}^{1}}(1)\right)$ for every fiber $f$ of $\pi$, it follows that $\left(q^{-1}(y), H_{q^{-1}(y)}\right)=\left(\mathbb{P}^{1} \times \mathbb{P}^{1}, \mathcal{O}_{\mathbb{P}^{1} \times \mathbb{P}^{1}}(1,1)\right)$, for every $y \in Y$. Hence $q_{\mid S}^{-1}(y)$ consists of the points at which two elements of $\left|\mathcal{O}_{\mathbb{P}^{1} \times \mathbb{P}^{1}}(1,1)\right|$ meet. This gives $d_{2}=K_{S}^{2}=2\left(-K_{Y}\right)^{2}=4$. Moreover for $E$ a smooth element of $\left|-K_{Y}\right|$ we get $K_{S} H_{S}=q_{\mid S}^{*}\left(-K_{Y}\right) H^{2}=q^{*}\left(-K_{Y}\right) H^{3}=p^{*} X_{E} H^{3}=\left(H_{p^{*} X_{E}}\right)^{3}$. Note that $H_{p^{*} X_{E}}$ is the tautological line bundle of $\mathcal{E}_{X_{E}}$. Hence, recalling (0.6.1), the last term is 
equal to $\left(c_{1}(\mathcal{E})^{2}-c_{2}(\mathcal{E})\right)_{X_{E}}$. Thus Corollary (4.2) shows that $K_{S} H_{S}=12$. Finally, to compute $\chi\left(\mathcal{O}_{S}\right)$, consider the exact sequence

$$
0 \rightarrow K_{Z} \rightarrow K_{Z}+H_{Z} \rightarrow K_{S} \rightarrow 0
$$

Recall that $h^{i}\left(K_{Z}\right)=0$ for $0 \leq i \leq 2$, since $Z$ is rational. Moreover $h^{i}\left(K_{Z}+H_{Z}\right)=0$ for $1 \leq i \leq 3$ by the Kodaira vanishing theorem. This gives $\chi\left(\mathcal{O}_{S}\right)=\chi\left(K_{S}\right)=$ $1+h^{0}\left(K_{S}\right)=1+h^{0}\left(K_{Z}+H_{Z}\right)$. On the other hand since $K_{Z}+H_{Z}=q_{\mid Z}^{*}\left(-K_{Y}\right)$ and $q_{\mid Z}: Z \rightarrow Y$ is a morphism with connected fibers, we have $\left(q_{\mid Z}\right)_{*} \mathcal{O}_{Z}=\mathcal{O}_{Y}$, and so $h^{0}\left(K_{Z}+H_{Z}\right)=h^{0}\left(-K_{Y}\right)=3$ by the projection formula. Therefore $\chi\left(\mathcal{O}_{S}\right)=4$. Putting all this information into (4.4.1) gives

$$
56 \geq(20-d) d .
$$

However, the corresponding equality has no integral solutions.

(4.5) Remark. Let $X, Y, \mathcal{E}$ be as in $(*)$. Then $(\operatorname{det} \mathcal{E})^{3} \geq 17+2(\operatorname{det} \mathcal{E}) c_{2}(\mathcal{E})$.

Proof. Let things be as in the proof of Lemma (4.4). Since $\left|H_{Z}\right|$ embeds $Z$ in $\mathbb{P}^{N}$ with $N \geq 7$ we have that $d=H^{4}=H_{Z}^{3} \geq N-3+1 \geq 5$. Thus (4.4.2) cannot be satisfied unless $d \geq 17$. Then the assertion follows recalling (0.6.2).

In fact we can say much more.

(4.6) Lemma. Let $X, Y, \mathcal{E}$ be as in $(*)$. Then $(\operatorname{det} \mathcal{E})^{3} \leq 42$. Moreover we have $(\operatorname{det} \mathcal{E}) c_{2}(\mathcal{E}) \leq 12$, and, if equality holds, then $(\operatorname{det} \mathcal{E})^{3}=42$.

Proof. Let $X=\mathbb{P}(\mathcal{V})$ and let $\xi$ be the tautological line bundle on $X$. From the relation

$$
\pi^{*}\left(-K_{Y}\right)=K_{X}+\operatorname{det} \mathcal{E}=-2 \xi+\pi^{*}\left(K_{Y}+\operatorname{det} \mathcal{V}\right)+\operatorname{det} \mathcal{E}
$$

we get $\operatorname{det} \mathcal{E}=2 \xi-\pi^{*}\left(2 K_{Y}+\operatorname{det} \mathcal{V}\right)$. Hence

$$
(\operatorname{det} \mathcal{E})^{3}=8 \xi^{3}-12 \xi^{2} \pi^{*}\left(2 K_{Y}+\operatorname{det} \mathcal{V}\right)+6 \xi \pi^{*}\left(2 K_{Y}+\operatorname{det} \mathcal{V}\right)^{2} .
$$

By using (0.6.1) and the Chern-Wu relation, this gives

$$
\begin{aligned}
(\operatorname{det} \mathcal{E})^{3} & =8(\operatorname{det} \mathcal{V})^{2}-8 c_{2}(\mathcal{V})-12 \operatorname{det} \mathcal{V}\left(2 K_{Y}+\operatorname{det} \mathcal{V}\right) \\
& +6(\operatorname{det} \mathcal{V})^{2}+24 K_{Y} \operatorname{det} \mathcal{V}+24 K_{Y}^{2} \\
& =2\left[(\operatorname{det} \mathcal{V})^{2}-4 c_{2}(\mathcal{V})\right]+48
\end{aligned}
$$

Suppose that $(\operatorname{det} \mathcal{V})^{2}>4 c_{2}(\mathcal{V})$. Then $\mathcal{V}$ is a Bogomolov unstable rank-2 bundle on $Y$. Hence there exists a zero-dimensional subscheme $\mathcal{Z}$ of $Y$ and line bundles $L$ and $M$ on $Y$ sitting in an exact sequence

$$
0 \rightarrow L \rightarrow \mathcal{V} \rightarrow M \otimes \mathcal{I}_{\mathcal{Z}} \rightarrow 0,
$$

such that $(L-M) A>0$ for any ample line bundle $A$ on $Y$ ( $\mathrm{Bo}$, see also $\mathrm{R}$ Theorem 1]). In particular $(L-M)\left(-K_{Y}\right)>0$. However, by Lemma (4.1) we know that $\mathcal{V}_{E}$ is a twist of a vector bundle $\mathcal{U}$ as in (0.7.1), for any smooth curve $E \in\left|-K_{Y}\right|$. So, restricting the exact sequence above to such a curve $E$ not meeting $\mathcal{Z}$ we should get that $\operatorname{deg} L_{E}-\operatorname{deg} M_{E}<0$, a contradiction. Therefore $(\operatorname{det} \mathcal{V})^{2}-4 c_{2}(\mathcal{V}) \leq 0$, hence (4.6.1) shows that

$$
(\operatorname{det} \mathcal{E})^{3} \leq 48
$$

To improve this inequality let us compute $(\operatorname{det} \mathcal{V})^{2}-4 c_{2}(\mathcal{V})$. Recall that $Y$ is $\mathbb{P}^{2}$ blown-up at seven points in general position. Let $E_{i} \subset Y(i=1, \ldots, 7)$ be the 
corresponding exceptional curves and let $\ell$ be the total transform of a line of $\mathbb{P}^{2}$. Since $\ell$ and the $E_{i}$ 's generate $\operatorname{Pic}(Y)$ we can write $\operatorname{det} \mathcal{V}=\sum_{i=1}^{7} \lambda_{i} E_{i}+\beta \ell$ for some integers $\lambda_{i}$ and $\beta$. Let $E \in\left|-K_{Y}\right|$ be a smooth curve. Recall that $\mathcal{V}_{E}$ is a twist of a vector bundle $\mathcal{U}$ as in (0.7.1) by Lemma (4.1), hence

i) $\operatorname{det} \mathcal{V}\left(-K_{Y}\right)$ is odd.

On the other hand, by Lemma (4.3) we know that

ii) $(\operatorname{det} \mathcal{V}) E_{i}$ is even for every $i=1, \ldots, 7$.

Intersecting $(\operatorname{det} \mathcal{V})$ with $-K_{Y}$ and with the $E_{i}$ 's, conditions i) and ii) show that $\sum_{i=1}^{7} \lambda_{i}+3 \beta$ is odd while $-\lambda_{i}$ is even, hence $\beta$ is odd. So we get the congruence

$$
(\operatorname{det} \mathcal{V})^{2}-4 c_{2}(\mathcal{V})=\sum_{i=1}^{7}\left(-\lambda_{i}\right)^{2}+9 \beta^{2}-4 c_{2}(\mathcal{V}) \equiv 1 \bmod 4
$$

Hence, recalling (4.6.1), we see that

$$
(\operatorname{det} \mathcal{E})^{3} \equiv 2 \bmod 8 .
$$

Then the first assertion follows from (4.6.2) and (4.6.3). The inequality $(\operatorname{det} \mathcal{E}) c_{2}(\mathcal{E})$ $\leq 12$ follows immediately taking into account Remark (4.5). Finally, the congruence (4.6.3) combined with Remark (4.5) again gives the last assertion.

(4.7) Now let us continue our proof to rule out case $(*)$. First note that

$$
h^{0}\left(\mathcal{E} \otimes \pi^{*} K_{Y}\right) \geq 1 .
$$

To see this let $E \in\left|-K_{Y}\right|$ be a smooth curve, and consider $X_{E}$ and the exact sequence

$$
0 \rightarrow \mathcal{E}\left(-X_{E}\right) \rightarrow \mathcal{E} \rightarrow \mathcal{E}_{X_{E}} \rightarrow 0 .
$$

Recall that $h^{0}(\mathcal{E}) \geq 9$ by Lemma (4.4). Then $h^{0}\left(\mathcal{E} \otimes \pi^{*} K_{Y}\right)=h^{0}\left(\mathcal{E}\left(-X_{E}\right)\right) \geq$ $9-h^{0}\left(\mathcal{E}_{X_{E}}\right)=1$, by the third assertion in Corollary (4.2).

Claim. Any nontrivial section of $\mathcal{E} \otimes \pi^{*} K_{Y}$ vanishes nowhere. Moreover we have that $(\operatorname{det} \mathcal{E}) c_{2}(\mathcal{E})=12$.

Proof. Let $s$ be such a section and let $t$ be its restriction to $X_{E}$ for $E$ a smooth curve in $\left|-K_{Y}\right|$. The exact sequence (4.1.1) gives, after a suitable twist,

$$
0 \rightarrow[\xi-f] \rightarrow\left(\mathcal{E} \otimes \pi^{*} K_{Y}\right)_{X_{E}} \rightarrow \xi \rightarrow 0 .
$$

Recall that $h^{0}(\xi-f)=0$ by (0.7.4). Hence $t$ maps injectively to the nonzero section of $\xi$, which vanishes on a section $\sigma$ of $X_{E}$. Hence, if $\operatorname{dim}\left(t^{-1}(0)\right)=1$, then $t$ vanishes on $\sigma$. Thus the injection $\mathcal{O}_{X_{E}} \rightarrow\left(\mathcal{E} \otimes \pi^{*} K_{Y}\right)_{X_{E}}$ defined by that section factors through an injection $\xi \rightarrow\left(\mathcal{E} \otimes \pi^{*} K_{Y}\right)_{X_{E}}$, hence there is an injection $\mathcal{O}_{X_{E}} \rightarrow\left(\mathcal{E} \otimes \pi^{*} K_{Y}\right)_{X_{E}} \otimes \xi^{-1}$. But this is impossible, since the latter has no sections, being isomorphic to $\left(\pi_{\mid X_{E}}^{*} \mathcal{U}\right)(-f)$, as shown by (4.1.4). It thus follows that $t$ can vanish at most at a finite set of points on $X_{E}$. On the other hand, recalling Lemma (4.1) and Corollary (4.2) we have

$$
c_{2}\left(\left(\mathcal{E} \otimes \pi^{*} K_{Y}\right)_{X_{E}}\right)=c_{2}\left(\mathcal{E}_{X_{E}}\right)+c_{1}\left(\mathcal{E}_{X_{E}}\right) \pi_{\mid X_{E}}^{*} K_{Y}=4+(2 \xi+3 f)(-2 f)=0 .
$$

Thus $s$ restricts to a section $t$ of $\left(\mathcal{E} \otimes \pi^{*} K_{Y}\right)_{X_{E}}$, which vanishes nowhere for a general $E \in\left|-K_{Y}\right|$. This shows that $\pi\left(s^{-1}(0)\right)$ is in the complement of $E$, which is an ample divisor on $Y$, hence it is a finite set. In other words, $s$ vanishes along a finite number, say $\delta$, of fibers of $X$. Now intersect $s^{-1}(0)$ with the ample divisor $\operatorname{det} \mathcal{E}$. 
Recalling the first assertion of Corollary $(4.2)$ and the fact that $(\operatorname{det} \mathcal{E})_{f}=\mathcal{O}_{\mathbb{P}^{1}}(2)$ for every fiber, we get

$$
\begin{aligned}
2 \delta=(\operatorname{det} \mathcal{E}) c_{2}\left(\mathcal{E} \otimes \pi^{*} K_{Y}\right) & =(\operatorname{det} \mathcal{E})\left(c_{2}(\mathcal{E})+(\operatorname{det} \mathcal{E}) \pi^{*} K_{Y}+\left(\pi^{*} K_{Y}\right)^{2}\right) \\
& =(\operatorname{det} \mathcal{E}) c_{2}(\mathcal{E})-16+4
\end{aligned}
$$

Since $\delta \geq 0$, this gives $(\operatorname{det} \mathcal{E}) c_{2}(\mathcal{E}) \geq 12$. Combining this inequality with Lemma $(4.6)$ gives $(\operatorname{det} \mathcal{E}) c_{2}(\mathcal{E})=12$, hence $\delta=0$. This shows that $s$ vanishes nowhere.

Now, due to the claim, the section $s$ defines an exact sequence

$$
0 \rightarrow \mathcal{O}_{X} \rightarrow \mathcal{E} \otimes \pi^{*} K_{Y} \rightarrow \operatorname{det}\left(\mathcal{E} \otimes \pi^{*} K_{Y}\right) \rightarrow 0 .
$$

Therefore, twisting by $-\pi^{*} K_{Y}$ and recalling that $K_{X}+\operatorname{det} \mathcal{E}=-\pi^{*} K_{Y}$, we get the exact sequence

$$
0 \rightarrow-\pi^{*} K_{Y} \rightarrow \mathcal{E} \rightarrow-K_{X} \rightarrow 0
$$

and so we conclude that $-K_{X}$ is very ample, as a quotient of $\mathcal{E}$. Now note that $(\operatorname{det} \mathcal{E})^{3}=42$ in view of the claim and Lemma (4.6). Recalling the first assertion in Corollary (4.2), we thus get

$$
\begin{aligned}
\left(-K_{X}\right)^{3} & =\left(\operatorname{det} \mathcal{E}+\pi^{*} K_{Y}\right)^{3} \\
& =(\operatorname{det} \mathcal{E})^{3}+3(\operatorname{det} \mathcal{E})^{2} \pi^{*} K_{Y}+3(\operatorname{det} \mathcal{E})\left(\pi^{*} K_{Y}\right)^{2} \\
& =42-48+12=6 .
\end{aligned}
$$

But this gives a contradiction, since $\left(X,-K_{X}\right)$ would appear in the classification of smooth projective varieties of degree 6 , which is not the case [I1]. Note that the same contradiction also follows from [SW], since $X$ would be a Fano bundle.

(4.8) Remark. In the course of disproving the existence of case $(*)$ we were led to the construction of a remarkable example: a threefold $X$ endowed with a non-very ample vector bundle $\mathcal{E}$ with the same invariants as those that arose in the proof. The existence of this example is the likely reason why so much work was required to show the nonexistence of case $(*)$. We would like to give the description of this pair $(X, \mathcal{E})$.

Let $Y$ be a Del Pezzo surface with $K_{Y}^{2}=2$ and let $\ell$ be the total transform of a general line of $\mathbb{P}^{2}$ under the blow-up $\eta: Y \rightarrow \mathbb{P}^{2}$ at seven points in general position. Note that $H^{1}\left(2 K_{Y}+\ell\right) \cong \mathbb{C}$. Let $\mathcal{V}$ be the rank-2 vector bundle on $Y$ defined by the unique nontrivial extension

$$
0 \rightarrow \mathcal{O}_{Y} \rightarrow \mathcal{V} \rightarrow \mathcal{O}_{Y}\left(-\ell-2 K_{Y}\right) \rightarrow 0 .
$$

Set $X:=\mathbb{P}(\mathcal{V})$, let $\pi: X \rightarrow Y$ be the induced projection and let $\xi$ denote the tautological line bundle on $X$. Note that given a smooth curve $E \in\left|-K_{Y}\right|$, the restriction to $E$ gives an isomorphism $H^{1}\left(Y, 2 K_{Y}+\ell\right) \stackrel{\cong}{\longrightarrow} H^{1}(E, \mathcal{M})$ where $\mathcal{M}$ is a line bundle of degree -1 on $E$. This says that $\mathcal{V}_{E}$ is isomorphic to a vector bundle $\mathcal{U}$ as in (0.7.1). Note that $K_{X}=-2 \xi+\pi^{*}\left(K_{Y}-\ell-2 K_{Y}\right)=-2 \xi-\pi^{*}\left(\ell+K_{Y}\right)$ by the canonical bundle formula. Thus, by applying Serre duality on $X$ we see that

$$
\begin{aligned}
H^{1}\left(-\pi^{*} K_{Y}+K_{X}\right) & =H^{1}\left(-2 \xi-\pi^{*}\left(\ell+2 K_{Y}\right)\right) \\
& \cong H^{2}\left(K_{X}+2 \xi+\pi^{*}\left(\ell+2 K_{Y}\right)\right) \\
& =H^{2}\left(\pi^{*} K_{Y}\right) \\
& \cong H^{2}\left(K_{Y}\right)=\mathbb{C} .
\end{aligned}
$$


Let $\mathcal{E}$ be the rank-2 vector bundle on $X$ defined by the unique nonsplit extension

$$
0 \rightarrow-\pi^{*} K_{Y} \rightarrow \mathcal{E} \rightarrow-K_{X} \rightarrow 0 .
$$

Of course we have $K_{X}+\operatorname{det} \mathcal{E}=\pi^{*}\left(-K_{Y}\right)$. Moreover it can be checked that

(1) $(\operatorname{det} \mathcal{E})^{3}=42$ and $(\operatorname{det} \mathcal{E}) c_{2}(\mathcal{E})=12$;

(2) $\mathcal{E}$ is spanned by global sections;

(3) $h^{0}(\mathcal{E})=9\left(\right.$ which in turn implies $\left.h^{0}\left(-K_{X}\right)=6\right)$;

(4) $\mathcal{E} \cong \pi^{*}\left(\mathcal{V} \otimes\left[K_{Y}+\ell\right]\right) \otimes \xi$.

This vector bundle $\mathcal{E}$ is not very ample of course. Actually it is easy to exhibit curves contracted by $-K_{X}$. For example, let $\sigma \in|\xi|$ be the section of $\pi: X \rightarrow Y$ corresponding to the surjection in (4.8.1). Then $\left(-K_{X}\right)_{\sigma}=2 \xi_{\sigma}+\pi_{\mid \sigma}^{*}\left(K_{Y}+\ell\right)=$ $\pi_{\mid \sigma}^{*}\left(-2 \ell-4 K_{Y}+K_{Y}+\ell\right)$. Therefore, $\left(-K_{X}\right)_{\sigma}$ can be identified with $-\ell-3 K_{Y}$ under the isomorphism $\pi_{\mid \sigma}: \sigma \rightarrow Y$. There are seven curves $e$ on $Y$ obtained as the proper transforms of cubics of $\mathbb{P}^{2}$ passing through the seven points blown-up under $\eta$, each with a node at one of the points. Note that for any $e$ from this set $\left(-\ell-3 K_{Y}\right) e=-3+3=0, e$ being a $(-1)$-curve.

\section{REFERENCES}

[ABW] M. Andreatta, E. Ballico, and J. A. Wiśniewski, Vector bundles and adjunction, Internat. J. Math. 3 (1992), 331-340. MR 93h:14031

[BS1] M. C. Beltrametti and A. J. Sommese, Comparing the classical and the adjunction theoretic definition of scrolls, Geometry of Complex Projective Varieties (A. Lanteri, M. Palleschi, and D. Struppa, eds.), Proc. Cetraro 1990, Mediterranean, Rende, 1993, pp. 5574. MR 94e: 14053

[BS2] - The Adjunction Theory of Complex Projective Varieties, Expositions in Mathematics, vol. 16, De Gruyter, Berlin - New York, 1995. MR 96f:14004

[BS3] - On the dimension of the adjoint linear system for threefolds, Ann. Sc. Norm. Sup. Pisa, IV 22 (1995), 1-24. MR 96e:14005

[Bo] F. Bogomolov, Holomorphic tensors and vector bundles on projective varieties, Math. USSR Isvestija 13 (1979), 499-555.

[Fu] W. Fulton, Intersection Theory, Ergebnisse der Math., vol. 2, Springer, 1984. MR 85k:14004

[Ha] R. Hartshorne, Ample vector bundles on curves, Nagoya Math. J. 43 (1971), 73-89. MR 45:1929

[I1] P. Ionescu, Embedded projective varieties of small invariants, Algebraic Geometry (L. Bădescu et al., eds.), Proc. Bucharest 1982, Springer, Berlin, 1984, pp. 142-186. MR 85m:14024

[I2] - Embedded projective varieties of small invariants, III, Algebraic Geometry (A. J. Sommese, A. Biancofiore, and E. L. Livorni, eds.), Proc. L'Aquila 1988, Springer, Berlin, Heidelberg, 1990, pp. 138-154. MR 91e:14014

[LM] A. Lanteri and H. Maeda, Ample vector bundle characterizations of projective bundles and quadric fibrations over curves, Higher Dimensional Complex Varieties (M. Andreatta and Th. Peternell, eds.), Proc. Trento 1994, De Gruyter, Berlin, New York, 1996, pp. 247-259. MR 98h:14051

[LMS] A. Lanteri, H. Maeda, and A. J. Sommese, Ample and spanned vector bundles of minimal curve genus, Arch. Math. 66 (1996), 141-149. MR 96k:14034

[LP] A. Lanteri and M. Palleschi, About the adjunction process for polarized algebraic surfaces, J. reine angew. Math. 352 (1984), 15-23. MR 86h:14028

[MS] H. Maeda and A. J. Sommese, Very ample vector bundles of curve genus two, Arch. Math. 79 (2002), 74-80. MR 2003f:14062

[Mo] S. Mori, Threefolds whose canonical bundles are not numerically effective, Ann. of Math. 116 (1982), 133-176. MR 84e:14032

[OSS] Ch. Okonek, M. Schneider, H. Spindler, Vector Bundles on Complex Projective Spaces, Progr. Math., vol. 3, Birkhäuser, Boston, 1980. MR 81b:14001 
[PSW] Th. Peternell, M. Szurek, and J. A. Wiśniewski, Fano manifolds and vector bundles, Math. Ann. 249 (1992), 151-165. MR 93h:14030

[R] M. Reid, Bogomolov's theorem $c_{1}^{2} \leq 4 c_{2}$, Proc. Internat. Symposium on Alg. Geom., Kyoto, 1977, pp. 623-642. MR 82b:14014

[Re] I. Reider, Vector bundles of rank 2 and linear systems on algebraic surfaces, Ann. of Math. 127 (1988), 309-316. MR 89e:14038

[S] A. J. Sommese, Submanifolds of abelian varieties, Math. Ann. 233 (1978), 229-256. MR 57:6524

[SV] A. J. Sommese and A. Van de Ven, On the adjunction mapping, Math. Ann. 278 (1987), 593-603. MR 88j:14011

[SW] M. Szurek and J. A. Wiśniewski, Fano bundles of rank 2 on surfaces, Compositio Math. 76 (1990), 295-305. MR 92e:14037

[YZ] Y.-G. Ye and Q. Zhang, On ample vector bundles whose adjunction bundles are not numerically effective, Duke Math. J. 60 (1990), 671-687. MR 91g:14040

Dipartimento di Matematica "F. Enriques", Università, Via C. Saldini 50, I-20133 Milano, Italy

E-mail address: lanteri@mat.unimi.it

Dipartimento di Matematica "F. Enriques", Università, Via C. Saldini 50, I-20133 Milano, Italy

E-mail address: palleschi@mat.unimi.it

Department of Mathematics, University of Notre Dame, Notre Dame, Indiana 465564618

E-mail address: sommese@nd.edu 